\title{
Complexities of gamma-ray line intensities from the lunar surface
}

\author{
Naoyuki Yamashita ${ }^{1}$, Nobuyuki Hasebe ${ }^{1}$, Takashi Miyachi ${ }^{1}$, Masanori Kobayashi ${ }^{2}$, Osamu Okudaira ${ }^{1}$, \\ Shingo Kobayashi ${ }^{1}$, Takeshi Ishizaki ${ }^{1}$, Kunitomo Sakurai ${ }^{1}$, Mitsuhiro Miyajima ${ }^{1}$, \\ Robert C. Reedy ${ }^{3}$, Claude d'Uston ${ }^{4}$, Sylvestre Maurice ${ }^{4}$, \\ and Olivier Gasnault ${ }^{4}$ \\ ${ }^{1}$ Research Institute for Science and Engineering, Waseda University, 3-4-1 Okubo, Shinjuku, Tokyo 169-8555 Japan \\ ${ }^{2}$ Nippon Medical School, 2-297-2 Kosugicho, Nakahara, Kawasaki, Kanagawa 211-0063 Japan \\ ${ }^{3}$ Institute of Meteoritics, University of New Mexico, Albuquerque, NM 87131-1126 USA \\ ${ }^{4}$ Centre d'Etude Spatiale des Rayonnements, 9 avenue Colonel Roche, B.P. 4346, 31028 Toulouse cedex 4, France
}

(Received March 19, 2007; Revised October 20, 2007; Accepted October 20, 2007; Online published April 9, 2008)

\begin{abstract}
Assuming different abundances of the Apollo lunar soil samples and the average spectrum of galactic cosmic ray protons, energy spectra of neutrons and gamma rays and emission rates of gamma-ray lines from major elements have been estimated by using the reviewed Monte Carlo simulation library Geant 4 and nuclear data. Previously, such libraries were not able to reproduce gamma-ray lines properly for the planetary application. Results clearly show that the emission rate of gamma rays heavily depends not only on the chemical abundance but also neutron flux within the lunar subsurface. While the intensities of gamma-ray lines are mostly proportional to elemental abundances, the intensity per unit elemental abundance can vary. Such a complex correlation is attributed to the change in neutron flux within the lunar subsurface and petrological restriction of elemental variation.
\end{abstract}

Key words: Gamma ray, neutron, lunar and planetary spectroscopy, moon, GRS, SELENE.

\section{Introduction}

Determining major and important minor and trace elements in the lunar surface is essential in the lunar and planetary science (Reedy et al., 1973; Reedy, 1978). Gamma-ray spectroscopy is suited for measuring the elemental composition in the lunar surface (Taylor, 1986). The Japanese lunar explorer SELENE will be launched in 2007 with a high precision gamma-ray spectrometer to survey the chemical abundance of the whole surface of the Moon (Hasebe et al., 2008; Sasaki et al., 2003). The spectrometer employing a $\mathrm{Ge}$ detector for the first time in the lunar exploration (Hasebe et al., 1999; Kobayashi et al., 2005) is going to uniquely identify the many important elements such as Fe, $\mathrm{Mg}, \mathrm{Ti}, \mathrm{Al}, \mathrm{Th}, \mathrm{K}, \mathrm{Ca}, \mathrm{U}, \mathrm{Si}, \mathrm{O}$ and possibly $\mathrm{H}$.

The characteristic gamma rays from the lunar surface are emitted from both decay of long-lived radioactive nuclides and nuclear interactions of galactic cosmic ray (GCR) particles with surface materials of the Moon (Reedy, 1978). Such interactions produce secondary neutrons, which lead to the emission of gamma rays mainly from nonelastic scattering reactions with fast neutrons and neutron capture reactions with thermal neutrons. The energies of the gamma rays are indicative of the nuclides responsible for the emission, and the intensities are related to their concentrations (Evans et al., 1993).

The energy regions of neutrons to be used in planetary nuclear spectroscopy are generally divided into three cate-

Copyright (C) The Society of Geomagnetism and Earth, Planetary and Space Sciences (SGEPSS); The Seismological Society of Japan; The Volcanological Society of Japan; The Geodetic Society of Japan; The Japanese Society for Planetary Sciences; TERRAPUB gories based on their interactions with matter; thermal $(<$ $0.4 \mathrm{eV})$, epithermal $(0.4 \mathrm{eV}<E<1 \mathrm{MeV})$, and fast (> $1 \mathrm{MeV})$. Fast neutrons excite surface nuclides by nonelastic scattering, which will be followed by emission of line gamma rays as a de-excitation process. Fast and epithermal neutrons are moderated to lower energies through elastic and nonelastic scattering to be in thermal equilibrium. Thermal neutrons are responsible for neutron capture reactions, which excites nuclides with their binding energies (Evans et al., 1993).

Production of neutrons and line gamma rays and their transport process in the lunar surface are needed to be investigated in detail to derive precise elemental abundance from gamma-ray observation (Masarik and Reedy, 1996).

Several ground experiments using an accelerator beam and thick targets have been executed to simulate the radiation environments on the Moon and planets (Englert et al., 1987; Brückner et al., 1987; Brückner et al., 1992; Yamashita et al., 2006). Such experiments should be complimented by Monte Carlo numerical simulations because the energy region of GCR cannot be covered by an accelerator, and it is very difficult to physically prepare target materials which can confine secondary neutrons that produce gamma rays (Yamashita et al., 2004a).

The simulation codes for the planetary application are required to be able to reproduce wide range of interaction from electromagnetic to hadronic ones. Good geometrical handling is necessary to reproduce a satellite and a detector system.

The role of the element atomic number on the production of the lunar fast neutrons has been investigated by Gasnault 
et al. (2000) using GEANT3, and gamma-ray spectroscopy data acquired by Lunar Prospector were modeled by Prettyman et al. (2006) using MCNPX.

However, Monte Carlo simulation codes for radiation such as MCNPX, GEANT3, Geant4 (Agostinelli et al., 2003) have trouble meeting all the requirements for high precision gamma-ray spectroscopy. Especially, none of the codes were able to reproduce energy spectra of gamma rays with all the line gamma rays induced by neutrons from the major elements correctly. It is essential to calculate both line gamma rays and continuum in spectra to estimate background gamma rays and sensitivity of a detector system. We have overcome such difficulties by reviewing the codes and nuclear data libraries using Geant4. Since SELENE is going to carry a highly precise gamma-ray spectrometer system on board, the production rates of neutrons and gamma rays were precisely estimated. Our calculations presented in this work have revealed a complex relationship between intensity of line gamma rays from the lunar surface and the elemental abundance.

\section{Process of Gamma-Ray Emission}

The lunar and planetary gamma rays are produced by decay of natural radioactive isotopes or interaction of high energy particles called galactic cosmic rays (GCR) with the surface material of planets. GCR propagate isotropically through interstellar space. Nuclear component of GCR consists of approximately $87 \%$ of protons, $12 \%$ of alpha particles, and $1 \%$ of heavier particles with charge $Z>3$ (Simpson, 1983). The energy spectra of GCR protons in the vicinity of the Moon are shown in Fig. 1. Below $\sim 10 \mathrm{GeV}$, GCR flux is modulated with the solar activity. The solar activity varies with a cycle of about 11 years, and therefore GCR flux changes with this cycle. The $4 \pi$ flux of GCR proton, $J$ [protons $/ \mathrm{cm}^{2} / \mathrm{s} / \mathrm{MeV}$ ] in the vicinity of the Earth is expressed as

$$
\begin{aligned}
J\left(E_{p}, \phi\right) & =C_{p} \frac{E_{p}\left(E_{p}+2 m_{p} c^{2}\right)\left(E_{p}+\chi+\phi\right)^{-2.65}}{\left(E_{p}+\phi\right)\left(E_{p}+2 m_{p} c^{2}+\phi\right)} \\
\chi & =780 \cdot \exp \left(-2.5 \cdot 10^{-4} \cdot E_{p}\right)
\end{aligned}
$$

where $E_{p}$ is the kinetic energy of the proton, $m_{p}$ is the proton mass, $c$ the velocity of light, $C_{p}$ is a normalization factor of $1.244 \times 10^{6}\left[\mathrm{~cm}^{-2} \mathrm{~s}^{-1} \mathrm{MeV}^{-1}\right]$ (Masarik and Reedy, 1994; Castagnoli et al., 1980). $\phi$ is the modulation parameter affected by solar activity and takes approximate values of $\phi=375 \mathrm{MeV}$ at solar minimum, $550 \mathrm{MeV}$ at solar average, or $950 \mathrm{MeV}$ at solar maximum (Reedy, 1987). The GCR proton has a maximum intensity at 400$800 \mathrm{MeV}$ and is modulated only at lower energies, as seen from Fig. 1.

\subsection{Neutron production}

The Moon, Mercury, Mars, and other satellites and asteroids without an atmosphere or with a thin atmosphere are directly exposed to GCR, and secondary neutrons are produced by nuclear interactions of GCR with surface materials (Feldman et al., 1999; Brückner and Masarik, 1997; Boynton et al., 2004). The surface of larger planets such as the Earth or Venus is shielded from the exposure of GCR by thick atmospheres. When an energetic proton enters a

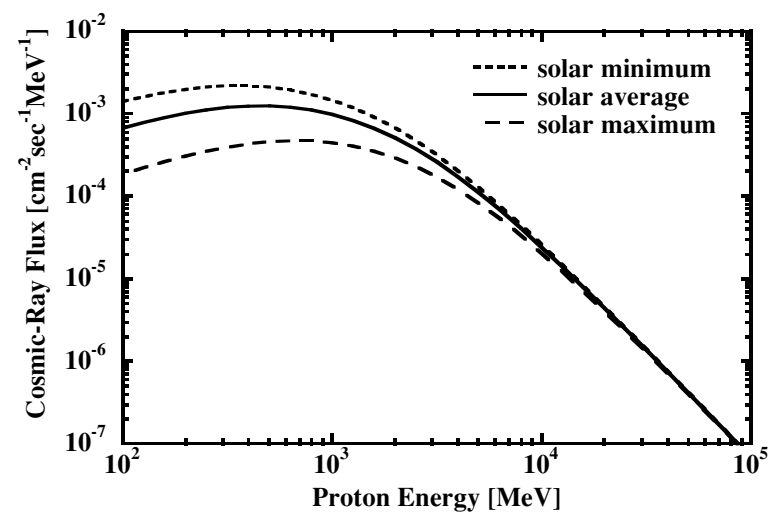

Fig. 1. Energy spectra of GCR protons at $1 \mathrm{AU}$ under three different solar conditions of the solar minimum, average, and maximum states.

Table 1. Energies of major line gamma rays expected from elements on the Moon. The superscripts for each energy indicate the following reactions that cause gamma-ray emission. c: radiative capture of neutrons, $\mathrm{n}$ : nonelastic scattering, $\mathrm{s}$ : spontaneous decay of naturally radioactive nuclides.

\begin{tabular}{cccccc}
\hline Elements & \multicolumn{5}{c}{ Gamma-ray energy $[\mathrm{MeV}]$} \\
\hline $\mathrm{H}$ & $2.223^{\mathrm{c}}$ & & & & \\
$\mathrm{O}$ & $6.129^{\mathrm{n}}$ & $4.438^{\mathrm{n}}$ & $5.269^{\mathrm{n}}$ & & \\
$\mathrm{Mg}$ & $1.369^{\mathrm{n}}$ & $1.809^{\mathrm{n}}$ & & & \\
$\mathrm{Al}$ & $1.014^{\mathrm{n}}$ & $2.211^{\mathrm{n}}$ & $0.844^{\mathrm{n}}$ & $7.724^{\mathrm{c}}$ & \\
$\mathrm{Si}$ & $1.779^{\mathrm{n}}$ & $3.539^{\mathrm{c}}$ & $4.934^{\mathrm{c}}$ & & \\
$\mathrm{S}$ & $2.230^{\mathrm{n}}$ & $5.421^{\mathrm{c}}$ & & & \\
$\mathrm{Ca}$ & $1.943^{\mathrm{c}}$ & $3.737^{\mathrm{n}}$ & $6.420^{\mathrm{c}}$ & & \\
$\mathrm{Ti}$ & $6.760^{\mathrm{c}}$ & $6.418^{\mathrm{c}}$ & $1.382^{\mathrm{c}}$ & $0.342^{\mathrm{c}}$ & $0.984^{\mathrm{n}}$ \\
$\mathrm{Fe}$ & $7.631^{\mathrm{c}}$ & $7.646^{\mathrm{c}}$ & $0.847^{\mathrm{n}}$ & & \\
$\mathrm{K}$ & $1.461^{\mathrm{s}}$ & & & & \\
$\mathrm{Th}$ & $2.615^{\mathrm{s}}$ & $0.911^{\mathrm{s}}$ & $0.239^{\mathrm{s}}$ & $0.583^{\mathrm{s}}$ & \\
$\mathrm{U}$ & $0.609^{\mathrm{s}}$ & $1.764^{\mathrm{s}}$ & $0.296^{\mathrm{s}}$ & & \\
\hline
\end{tabular}

thin target material, thin target yield of a secondary neutron $\sigma(p, n x)$ is defined as

$$
\sigma(p, n x)=\sigma_{\text {nonela }} \cdot M_{n}
$$

where $M_{n}$ is multiplicity indicating number of neutrons emitted per interaction, and $\sigma_{\text {nonela }}$ is nonelastic scattering cross section. As a result, neutrons with wide energy distribution are produced within the subsurface of a planet. The neutrons produced have energies of about $1-20 \mathrm{MeV}$ and higher and are called fast neutrons.

Such neutrons then interact with surface materials and cause nuclear excitations. Fast neutrons above $\sim 1 \mathrm{MeV}$ excite a nuclide by nonelastic scattering, in which part of kinetic energy of neutron is used to raise the energy level of the nuclide from the ground state to an excited state. Scattering reactions lower the energies of neutrons, and many neutrons are slowered to thermal energies $(\sim 0.02 \mathrm{eV})$. Some elements have high cross section for radiative capture reaction to thermal neutrons in which the binding energy of a neutron, $\sim 8 \mathrm{MeV} /$ nucleon in average, excites a nuclide that captures the neutron. 
Table 2. Abundances and densities of the lunar surface models used in the calculations after McKay et al. (1991). The abundances are expressed in $\mathrm{wt} \%$ in form of oxides and the densities in $\mathrm{g} / \mathrm{cm}^{3}$.

\begin{tabular}{|c|c|c|c|c|c|c|c|}
\hline & FAN & Apollo11 & Apollo12 & Apollo14 & Apollo15 & Apollo16 & Apollo17 \\
\hline Density & 3.11 & 3.27 & 3.21 & 3.13 & 3.27 & 3.14 & 3.24 \\
\hline $\mathrm{SiO}_{2}$ & 45.8 & 42.2 & 46.3 & 48.1 & 46.8 & 45.0 & 43.2 \\
\hline $\mathrm{TiO}_{2}$ & & 7.8 & 3 & 1.7 & 1.4 & 0.54 & 4.2 \\
\hline $\mathrm{Al}_{2} \mathrm{O}_{3}$ & 28.3 & 13.6 & 12.9 & 17.4 & 14.6 & 27.3 & 17.1 \\
\hline $\mathrm{Cr}_{2} \mathrm{O}_{3}$ & & 0.3 & 0.34 & 0.23 & 0.36 & 0.33 & 0.33 \\
\hline $\mathrm{FeO}$ & 4.8 & 15.3 & 15.1 & 10.4 & 14.3 & 5.1 & 12.2 \\
\hline $\mathrm{MnO}$ & & 0.2 & 0.22 & 0.14 & 0.19 & 0.3 & 0.17 \\
\hline $\mathrm{MgO}$ & 3.9 & 7.8 & 9.3 & 9.4 & 11.5 & 5.7 & 10.4 \\
\hline $\mathrm{CaO}$ & 17.0 & 11.9 & 10.7 & 10.7 & 10.8 & 15.7 & 11.8 \\
\hline $\mathrm{Na}_{2} \mathrm{O}$ & 0.29 & 0.47 & 0.54 & 0.7 & 0.39 & 0.46 & 0.4 \\
\hline $\mathrm{K}_{2} \mathrm{O}$ & & 0.16 & 0.31 & 0.55 & 0.21 & 0.17 & 0.13 \\
\hline $\mathrm{P}_{2} \mathrm{O}_{3}$ & & 0.05 & 0.4 & 0.51 & 0.18 & 0.11 & 0.12 \\
\hline Total & 100.09 & 99.78 & 99.11 & 99.83 & 100.73 & 100.71 & 100.05 \\
\hline
\end{tabular}

\subsection{Gamma-ray production}

The excited nuclides release energy to be back in the ground state by the emission of gamma rays, whose energies are determined by the difference of energy levels. Since energy levels are discrete and characteristic of a nuclide, the energies of the gamma rays are indicative of the nuclide that emitted them (Reedy, 1978).

The target elements of planetary gamma-ray spectroscopy that are induced by GCR include $\mathrm{Fe}, \mathrm{Mg}, \mathrm{Ti}, \mathrm{Al}$, $\mathrm{Ca}, \mathrm{Si}, \mathrm{O}$ and possibly $\mathrm{H}$. The energies of major line gamma rays from these elements are listed in approximate order of decreasing intensity in Table 1 (Reedy and Frankle, 2002; Firestone, 1999).

The intensity of line gamma rays depends on their abundance and reaction cross section of neutron inelastic scattering or neutron capture, if neutron flux is constant all over the target planet. However, sensitivity and calibration of gamma-ray intensity to elemental abundance necessarily vary from region to region because the neutron flux itself is a function of elemental abundance. Therefore, Monte Carlo simulation of neutrons and gamma rays in the planetary subsurface is essential.

\section{Calculation}

In order to numerically simulate neutron and gamma-ray production and their transport on the Moon, Monte Carlo library Geant 4 release 8.0.p01 has been employed. The lunar surface is modeled as a $20 \times 20 \times 20 \mathrm{~m}^{3}$ cube of $3.1-3.3 \mathrm{~g} / \mathrm{cm}^{3}$ densities with the composition of ferroan anorthosite (FAN) or lunar returned samples collected at Apollo landing sites (McKay et al., 1991). FAN is a rock type found in Apollo samples and considered as a main constitute of the surface of the Moon in the highland regions. The elemental composition and densities of FAN and the samples used in the calculations are shown in form of oxides in Table 2.

The incident particles are protons with monochromatic or GCR spectrum energies, isotropically irradiating to the center of the model cube. Most calculations assume the solar average condition, which is similar to when SELENE executes observation above the Moon, except the comparison of calculation results with observations by Lunar Prospec-

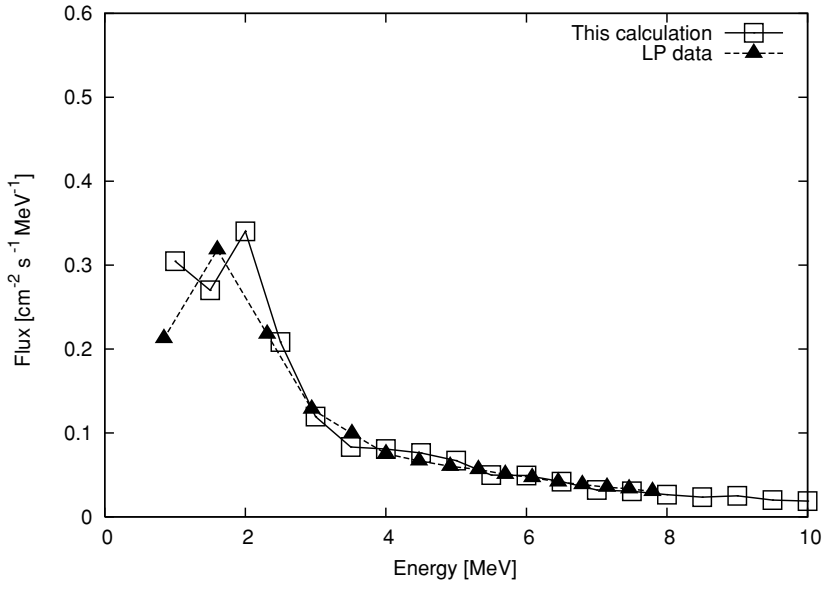

Fig. 2. Comparison of energy spectra of lunar fast neutrons between calculation and observation.

tor.

In order to take GCR alpha particles into account, gamma-ray production rate is multiplied by 1.4. The effect of alpha particles on the production rate of gamma rays is discussed in previous works such as Masarik and Reedy (1994), Masarik and Reedy (1996), and Mckinney et al. (2006), and will not be discussed in this study any further.

In order to validate the neutron calculations, the Geant4 results using the FAN target were compared with fast neutron fluxes observed by the Lunar Prospector Neutron Spectrometer (Maurice et al., 2000). In this comparison, the condition of the solar activity was coordinated with that during the observation in 1998, $\phi=346 \mathrm{MeV}$ (Mckinney et al., 2006). The observational data were quoted from Gasnault $e t$ al. (2000). The calculated and observed spectra of fast neutrons are shown in Fig. 2. A good agreement was obtained in the energy region of above $\sim 2 \mathrm{MeV}$, which benchmarked our neutron calculations based on Geant 4 with the observation results as well as previous calculations using GEANT3 (Gasnault et al., 2000).

The disagreement at lower energies can be attributed to the difference between the average composition of the Moon and that of FAN used in the calculation and changes 


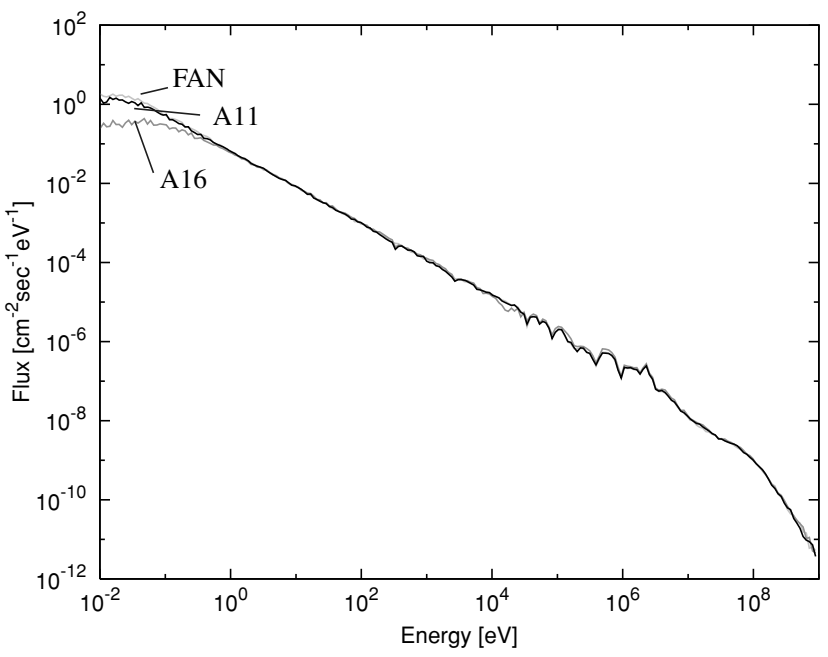

Fig. 3. Energy spectra of neutrons expected to emerge from the surface with the assumed compositions.

in detection efficiency or threshold of the spectrometer.

Our calculations of gamma-ray lines have also been verified with results from charged particle accelerator experiments (Yamashita et al., 2003; Yamashita et al., 2006) and reactor neutron beam experiments (Yamashita et al., 2004b).

\section{Results and Discussion}

\subsection{Neutron emission and transport}

The energy spectra of neutrons that leak from the lunar surface have been calculated and are shown in Fig. 3. The Apollo 11 site was selected as a representative mare region and the Apollo 16 site as a representative highland region for comparison. The spectrum of FAN region was shown because it is considered as a pure highland case and the contribution of crater ejecta from mare regions and other rocks can be eliminated. Large differences in neutron flux are seen in thermal energy region, while relatively small difference is seen in epithermal and fast energy regions. When considering thermal neutron fluxes, it is necessary to consider the neutron production, and moderation of higher energy neutrons, and the absorption of thermal neutrons.

Fast neutron fluxes do vary within the range of approximately $20 \%$ as depending on the elemental abundance of the target material. The detailed discussion on the flux and the composition, especially Fe and Ti contents, will be made in the following section.

The calculated results of neutron fluxes within the lunar subsurface are shown in Fig. 4. As seen from the figure, neutron fluxes have a peak depth within the subsurfaces, total neutron fluxes at about $120 \mathrm{~g} / \mathrm{cm}^{2}$, fast neutron fluxes at about $85 \mathrm{~g} / \mathrm{cm}^{2}$, and thermal neutron fluxes at about $150 \mathrm{~g} / \mathrm{cm}^{2}$ regardless of the abundances. However, changes in peak depth of neutron flux must contribute to the variation of line gamma-ray leakage flux because the upper layer of the surface itself shields line gamma rays produced at greater depths.

\subsection{Gamma-ray emission}

Since there exists a strong neutron flux within the subsurface of the Moon, energy spectra of gamma rays that

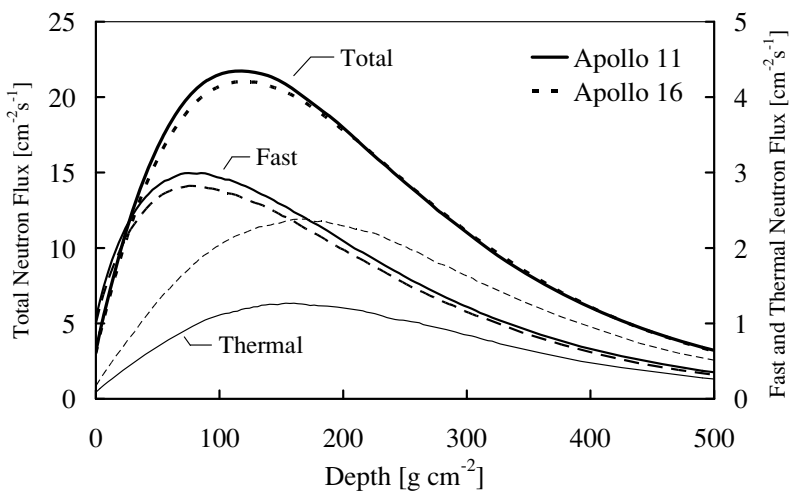

Fig. 4. Distribution of calculated neutron flux within the subsurface of the Moon. Note that the fast $(>1 \mathrm{MeV})$ and thermal $(<0.4 \mathrm{eV})$ neutron fluxes are denoted with the right vertical axis. Epithermal $(0.4 \mathrm{eV}$ $<E<1 \mathrm{MeV}$ ) neutrons account for the majority of the total neutron fluxes. The fluxes at Apollo 11 site are presented with solid lines and the fluxes at Apollo 16 site are presented with dashed lines.

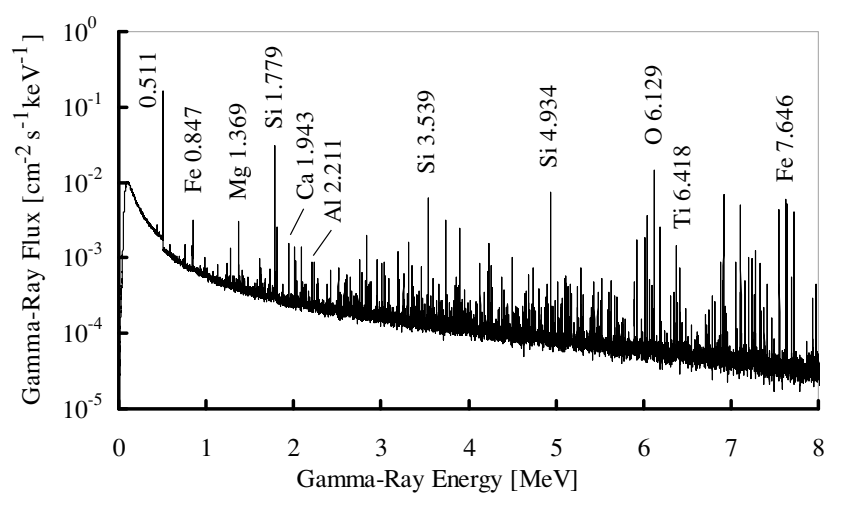

Fig. 5. Energy spectrum of gamma rays expected to emerge from the Apollo 16 site. Strong line gamma rays are labelled with their energies and nuclides.

emerge from the lunar surface are expected to have various lines from the major elements on top of a strong continuum. However, previous Monte Carlo calculations using codes such as Geant4, LAHET Code System (LCS), HERMES, MCNPX, or PHITS were unable to satisfactorily reproduce such line gamma rays for the application of the planetary gamma-ray spectroscopy, especially those from neutron capture reactions and many of those originated in inelastic scattering of neutrons. This inconsistency was overcome by introducing new nuclear data such as inelastic scattering cross sections of neutrons from JENDL-3.3 (Shibata et al., 2002) and branching ratios of nuclides after neutron capture reactions (Reedy and Frankle, 2002) to the calculation code. The nuclear reaction rates were calculated by using such evaluated nuclear data and neutron fluxes, as verified in Fig. 2, to derive gamma-ray emission rate.

As a result, it has become possible to calculate expected gamma-ray lines from major elements with continuum. In Fig. 5, calculated energy spectrum of gamma rays from the surface with the average soil compositions of the Apollo 16 site is shown. The peaks in the spectrum are labelled with their energies in $\mathrm{MeV}$ and elements from which the gamma ray was emitted. The spectrum has both neutron capture gamma-ray lines and inelastic gamma-ray lines. Contribu- 


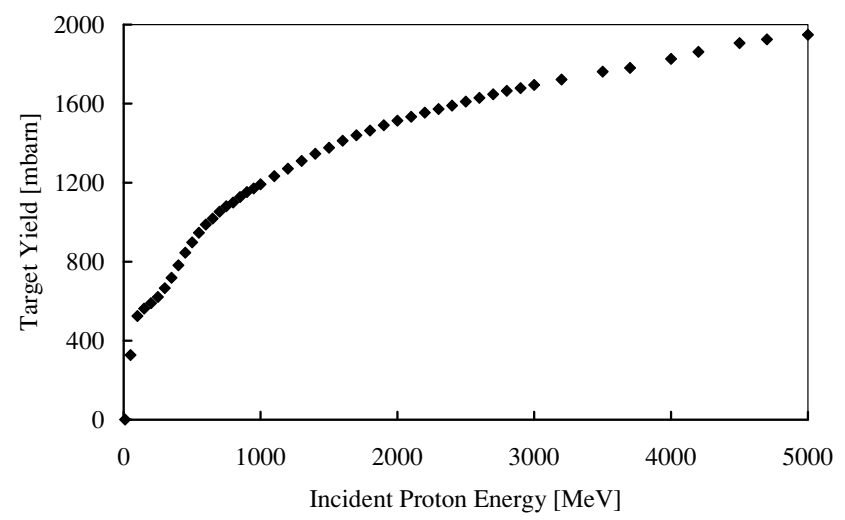

Fig. 6. Thin target yield of ${ }^{27} \mathrm{Al}$ to produce secondary neutrons with protons as a function of incident energies.

tions from natural radioactive nuclides such as ${ }^{40} \mathrm{~K},{ }^{232} \mathrm{Th}$, and ${ }^{238} \mathrm{U}$ are not taken into account, since they are not neutron-induced gamma-ray sources.

\subsection{Neutron production}

In order to study the relationship between the gamma-ray line intensities and the elemental abundances, the production of neutrons by GCR protons within the subsurfaces of the modeled lunar sites was investigated. Such studies are essential when interpreting orbital gamma-ray data to derive elemental compositions.

4.3.1 Yield Target yield is defined as production cross section of a particle times multiplicity, as expressed in Eq. (2).

According to experiments, the nonelastic cross section of high energy protons above $2 \mathrm{GeV}$ is expressed in form of a semi-empirical equation of

$$
\sigma_{\text {H.E. }}=45 A^{0.7}[1+0.016 \sin (5.3-2.63 \ln A)]
$$

as a function of atomic mass $A$ of the target nuclide (Letaw et al., 1983; Silberberg et al., 1998). Equation (3) gives $\sim 453$ mbarn for ${ }^{27} \mathrm{Al}$ and is consistent with the calculation of this work.

Yield of secondary particles increases monotonically with the proton's energy.

The calculated yield of $\mathrm{Al}$ is shown in Fig. 6. However, considering the energy spectrum of GCR, which decreases as a function of $E^{-2.75}$ at high energies (Derbina et al., 2005), it follows that the contribution to the production of secondary neutrons is the most effective at a few $\mathrm{GeV}$ region. Therefore, incident proton energy of $3 \mathrm{GeV}$ is employed as a representative energy of GCR for neutron production process in the following discussion.

4.3.2 Neutron multiplicity and yields of major elements The multiplicity of secondary neutrons and yields by major elements calculated by Geant 4 are shown in Fig. 7 when the incident energy of proton is at $3 \mathrm{GeV}$. The lightest element $\mathrm{O}$ has a multiplicity of $\sim 2.5$ while heavier elements of $\mathrm{Ti}$ has that of $\sim 6.6$ and $\mathrm{Fe} \sim 7.2$. Among elements that constitute the lunar surface, Fe and Ti produce secondary neutrons the most.

Figure 7 can be explained by the facts that the nonelastic cross section of neutrons at energies of higher than $\sim 3 \mathrm{GeV}$ is determined by geometrical cross section of elements and

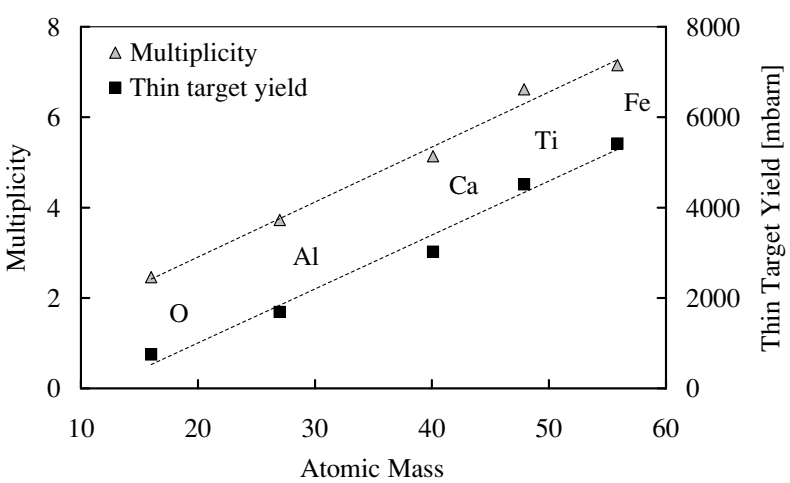

Fig. 7. Multiplicity for producing secondary neutrons and thin target yield of major elements of the Moon with $3 \mathrm{GeV}$ protons.

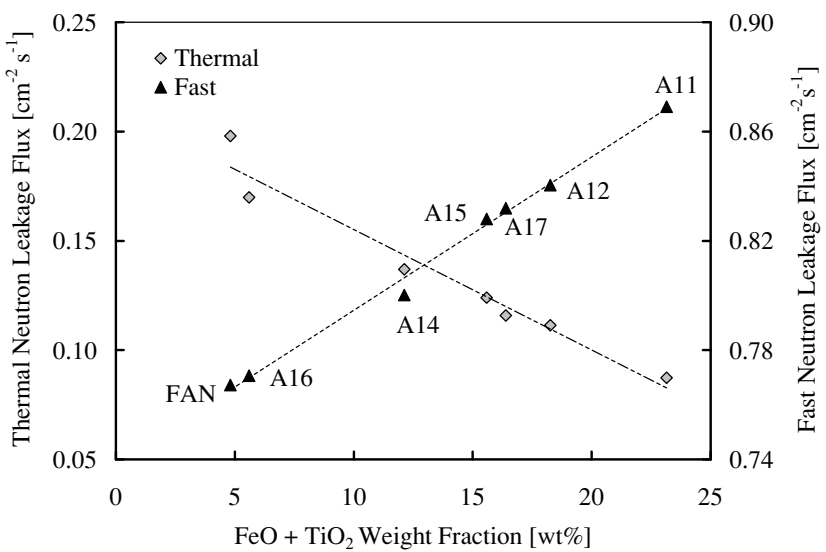

Fig. 8. Leakage fluxes of fast and thermal neutrons as a function of the weight fraction of $\mathrm{FeO}$ and $\mathrm{TiO}_{2}$.

is independent of incident proton energies, as expected from Eq. (3).

The change in multiplicity, and therefore neutron yields of major elements, indicates that the flux of secondary neutrons, the source of line gamma rays, is already dependent on the elemental composition and should be normalized when deriving elemental abundance from gamma-ray data.

It also shows a linear relationship of the multiplicity with the atomic mass, which was used by Gasnault et al. (2001) to derive an average atomic mass map of the lunar surface.

\subsection{Neutron flux and the effect of heavy elements}

In order to estimate the change in neutron flux by elemental composition, leakage neutron fluxes were calculated when the average flux of GCR protons for a solar cycle irradiated targets that have various abundances of FAN and the Apollo landing sites. The fluxes of thermal and fast neutrons were plotted separately as a function of composition of iron and titanium oxides in Fig. 8.

Since hydrogen is an effective moderator of neutrons, the existence of hydrogen within the subsurface of the Moon could cause variations in the neutron fluxes (Lawrence et al., 2006) as well as intensities of line gamma rays (Brückner and Masarik, 1997). However, the presence of hydrogen is only suggested at limited areas in the polar regions with permanent shade (Feldman et al., 2001), and such an effect is considered as negligible on most of the 


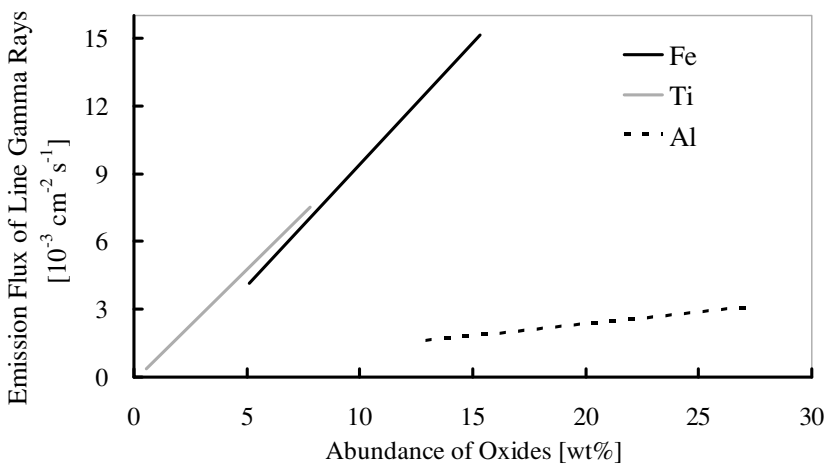

Fig. 9. Variation of line gamma-ray fluxes from ${ }^{27} \mathrm{Al}$ at $2.211 \mathrm{MeV},{ }^{56} \mathrm{Fe}$ at $0.847 \mathrm{MeV}$, and ${ }^{48} \mathrm{Ti}$ at $0.984 \mathrm{MeV}$ emerging from the modeled lunar sites. The fluxes are plotted as a function of the abundances of their metal oxides, $\mathrm{FeO}, \mathrm{TiO}_{2}$, and $\mathrm{Al}_{2} \mathrm{O}_{3}$.

surface of the Moon.

It is shown in Fig. 8 that the flux of fast neutron, responsible for inelastic scattering gamma-ray lines, has a linear dependence on the weight fraction of Fe and Ti. Because of the high multiplicities of these elements, regions rich in $\mathrm{Fe}$ and Ti such as the Apollo 11 or 12 landing sites, the mare regions on the Moon, are expected to have 15-20\% higher fast neutron fluxes than that for regions rich in $\mathrm{Al}$ such as the Apollo 16 landing site or FAN, the typical highland regions (Gasnault et al., 2000).

Thermal neutrons, responsible for neutron capture gamma-ray lines, decrease as the weight fraction of the heavy elements increases in spite of the increased fast neutron flux. Since both Fe and Ti have very high neutron capture cross section (Reedy and Frankel, 2002) of 2.60 barn and 6.10 barn compared to that of $\mathrm{Al}(0.23$ barn) or $\mathrm{Ca}$ ( 0.43 barn), more thermal neutrons are captured by Fe and Ti than $\mathrm{Al}$ and $\mathrm{Ca}$ before they escape from the surface in the mare regions, as seen in Fig. 8.

\subsection{Intensities of inelastic scattering gamma rays}

The fluxes of line gamma rays produced by inelastic scattering of neutrons with ${ }^{56} \mathrm{Fe}$ at $0.847 \mathrm{MeV},{ }^{48} \mathrm{Ti}$ at $0.984 \mathrm{MeV},{ }^{27} \mathrm{Al}$ at $2.211 \mathrm{MeV}$ were calculated with various composition of Apollo sites and are plotted as a function of their oxide abundances in Fig. 9. The Fe and Ti gammaray line intensities follow the similar law of dependence on their abundances because Fe and Ti happen to have similar inelastic cross sections for production of the lines and similar gamma-ray energies, causing attenuation coefficients to be close.

In general, the intensities show a correlation with the composition. Indeed, as seen from Fig. 9, Fe and Ti gammaray fluxes and their abundances correlate with steep positive slopes. However, Al gamma-ray fluxes correlate with a gentle slope.

Such difference is attributed to the change in fast neutron flux caused by a petrological restriction on the existence of rock forming minerals.

\subsection{Petrological restriction of elemental abundance}

When compared to their abundance variations, the variation of Fe and Ti gamma-ray line intensities are enhanced, while that of $\mathrm{Al}$ line intensities are reduced. When analyz- ing stone targets like the surface of the Moon, one should pay attention not only to the element that emits gamma rays but also other elements that have large contributions to production of neutrons which induce the gamma-ray emission. On the Moon, elements are contained as rock forming minerals such as plagioclase which has a high abundance of $\mathrm{Al}$ or mafic minerals such as olivine, pyroxene and ilumenite which have a high abundance of $\mathrm{Fe}$ and $\mathrm{Ti}$. In general, the composition of such minerals has a reciprocal relationship in a rock. For example, basalt and gabbro mainly consist of olivine and pyroxene, and rhyolite and granite mainly consist of plagioclase. Therefore, changes in the abundance of a single element is not independent of that of other elements, indicating increasing amount of plagioclase, rich in $\mathrm{Al}$, means decreasing amount of mafic minerals, rich in $\mathrm{Fe}$ and Ti. In the calculations of this work, changes in the $\mathrm{Al}$ content were considered to be overwhelmed by those in $\mathrm{Fe}$ and $\mathrm{Ti}$ which significantly affects the fast neutron flux as discussed in Section 4.4. As a result, while the absolute Al gamma-ray flux increased with its abundance, the ratio of the $\mathrm{Al}$ flux to $\mathrm{Al}$ abundance decreased.

\section{Conclusions}

By using the reviewed Geant4 library and nuclear data, the detailed calculation of neutrons and gamma-ray lines together with continua in energy spectra became possible. Such numerical calculations are essential to determine the relationship between the abundances and the intensities of gamma-ray lines for planetary nuclear spectroscopy and can be applied to analyses of cosmogenic nuclides in meteorites or shield design for accelerator facilities.

The total emission rate of line gamma rays is closely related to the change in neutron flux produced in the subsurface of the Moon. It is concluded that the concentration of heavy elements such as Fe and Ti among the major elements of the lunar surface material is directly determined from the emission rate of their line gamma rays, while that of light elements such as Al should be determined with consideration of change in the abundance of heavier elements on the Moon. Therefore, while gamma-ray spectroscopy itself reveals important information regarding the chemical composition of the lunar surface, it is important that gamma-ray data are complemented by those from a neutron spectrometer, which SELENE does not carry, such as those measured by Lunar Prospector and expected by Lunar Reconnaissance Orbiter, or that careful and repeated computer simulations should be made for determination of elemental abundance with high reliability.

\section{References}

Agostinelli, S. et al., Geant4-a simulation toolkit, Nucl. Inst. and Methods in Phys. Res., A506, 250-303, 2003.

Boynton, W. V., W. C. Feldman, I. G. Mitrofanov, L. G. Evans, R. C. Reedy, S. W. Squyres, R. Starr, J. I. Trombka, C. d'Uston, J. R. Arnold, P. A. J. Englert, A. E. Metzger, H. Wanke, J. Brückner, D. M. Drake, C. Shinohara, C. Fellows, D. K. Hamara, K. Harshman, K. Kerry, C. Turner, M. Ward, H. Barthe, K. R. Fuller, S. A. Storms, G. W. Thornton, J. L. Longmire, M. L. Litvak, and A. K. Ton'chev, The Mars Odyssey Gamma-Ray Spectrometer Instrument Suite, Space Sci. Rev., 110, 3783, 2004.

Brückner, J. and J. Masarik, Planetary gamma-ray spectroscopy of the surface of Mercury, Planet. Space Sci., 45, No. 1, 1997. 
Brückner, J., H. Wänke, and R. C. Reedy, Neutron-induced gamma ray spectroscopy: Simulations for chemical mapping of planetary surfaces, J. Geophys. Res., 92, E603, 1987.

Brückner, J., U. Fabian, A. Patnaik, H. Wänke, P. Cloth, G. Dagge, V. Druke, D. Filges, P. A. J. Englert, D. M. Drake, R. C. Reedy, and B. Parlier, Simulation experiments for planetary gamma-ray Spectroscopy by means of thick target high-energy proton irradiations, Lunar and Planetary Sci. Conf., XXIII, 169, 1992.

Castagnoli, G. et al., Solar modulation effects in terrestrial production of carbon-14, Radiocarbon, 22, 1980.

Derbina, V. A., V. I. Galkin, M. Hareyama, Y. Hirakawa, Y. Horiuchi, M. Ichimura, N. Inoue, E. Kamioka, T. Kobayashi, V. V. Kopenkin, S. Kuramata, A. K. Managadze, H. Matsutani, N. P. Misnikova, R. A. Mukhamedshin, S. Nagasawa, R. Nakano, M. Namiki, M. Nakazawa, H. Nanjo, S. N. Nazarov, S. Ohata, H. Ohtomo, V. I. Osedlo, D. S. Oshuev, P. A. Publichenko, I. V. Rakobolskaya, T. M. Roganova, C. Saito, G. P. Sazhina, H. Semba, T. Shibata, D. Shuto, H. Sugimoto, R. Suzuki, L. G. Sveshnikova, V. M. Taran, N. Yajima, T. Yamagami, I. V. Yashin, E. A. Zamchalova, G. T. Zatsepin, and I. S. Zayarnaya, CosmicRay Spectra and Composition in the Energy Range of 10-1000 TeV per Particle Obtained by the RUNJOB Experiment, Astrophys J., 628, L41-L44, 2005.

Englert, P., R. C. Reedy, and J. R. Arnold, Thick-target bombardments with high energy charged particles: Experimental improvements and spatial distribution of low-energy secondary neutrons, Nucl. Inst. and Methods in Phys. Res. A, 262, 496, 1987.

Evans, L. G., R. C. Reedy, and J. I. Trombka, Introduction to Planetary Remote Sensing Gamma Ray Spectroscopy, in Remote Geochemical Analysis: Elemental and Mineralogical Composition, edited by C. M. Pieters and P. A. J. Englert, 594 pp, Cambridge University Press, Cambridge, 1993.

Feldman, W. C., B. L. Barraclough, K. R. Fuller, D. J. Lawrence, S. Maurice, M. C. Miller, T. H. Prettyman, and A. B. Binder, The Lunar Prospector gamma-ray and neutron spectrometers, Nucl. Inst. Methods, A422, 562, 1999.

Feldman, W. C., S. Maurice, D. J. Lawrence, R. C. Little, S. L. Lawson, O. Gasnault, R. C. Wiens, B. L. Barraclough, R. C. Elphic, T. H. Prettyman, J. T. Steinberg, and A. B. Binder, Evidence for water ice near the lunar poles, J. Geophys. Res., E106, 23231-23252, 2001.

Firestone, R. B., Tables of Isotopes Data, in Table of Isotopes Eighth Edition, edited by R. B. Firestone and V. S. Shirley, 2877 pp, WileyInterscience Publication, New York, 1999.

Gasnault, O., C. d'Uston, W. C. Feldman, and S. Maurice, Lunar fast neutron leakage flux calculation and its elemental abundance dependence, J. Geophys. Res., E105, 4263-4271, 2000.

Gasnault, O., W. C. Feldman, S. Maurice, I. Genetay, C. d'Uston, T. H. Prettyman, and K. R. Moore, Composition from fast neutrons: application to the Moon, Geophys. Res. Lett., 28, 3797-3800, 2001.

Hasebe, N., E. Shibamura, T. Atarashiya, T. Doke, C. d'Uston, M. Grande, T. Kashiwagi, J. Kikuchi, M. Kobayashi, K. Kubo, S. Mitani, K. Mori, K. Narasaki, K. Nomi, T. Takashima, R. Tanibata, and R. C. Reedy, Gamma-ray spectrometer for Japanese lunar polar orbiter, Adv. Space Res., 23, 1837-1840, 1999.

Hasebe, N., E. Shibamura, T. Miyachi, T. Takashima, M. Kobayashi, O. Okudaira, N. Yamashita, S. Kobayashi, T. Ishizaki, K. Sakurai, M. Miyajima, M. Fujii, K. Narasaki, S. Takai, K. Tsurumi, H. Kaneko, M. Nakazawa, K. Mori, O. Gasnault, S. Maurice, C. d'Uston, R. C. Reedy, and M. Grande, Gamma-ray spectrometer (GRS) for lunar polar orbiter SELENE, Earth Planets Space, 60, this issue, 299-312, 2008.

Kobayashi, M., N. Hasebe, T. Hiramoto, T. Miyachi, S. Murasawa, H. Okada, O. Okudaira, N. Yamashita, A. Berezhnoy, and E. Shibamura, Germanium detector with Stirling cryocooler for lunar gamma-ray spectroscopy, Nucl. Inst. Methods in Phys. Res., A548, 401-410, 2005.

Lawrence, D. J., W. C. Feldman, R. C. Elphic, J. J. Hagerty, S. Maurice, G. W. McKinney, and T. H. Prettyman, Improved modeling of Lunar Prospector neutron spectrometer data: Implications for hydrogen deposits at the lunar poles, J. Geophys. Res., 111, E08001, 2006.

Letaw, J. R., R. Silberberg, and C. H. Tsao, Proton-nucleus total inelastic cross sections - an empirical formula for E greater than $10 \mathrm{MeV}$, The Astrophys. J. Supplement Series, 51, 271-276, 1983.

Masarik, J. and R. C. Reedy, Effects of bulk composition on nuclide pro- duction processes in meteorites, Geochim. Cosmochm. Acta, 58, 5307$5317,1994$.

Masarik, J. and R. C. Reedy, Gamma ray production and transport in Mars, J. Geophys. Res., E101, 18891-18912, 1996.

Maurice, S., W. C. Feldman, D. J. Lawrence, R. C. Elphic, O. Gasnault, C. d'Uston, I. Genetay, and P. G. Lucey, High-energy neutrons from the Moon, J. Geophys. Res., E105, 20365-20375, 2000.

McKay, D. S., G. Heiken, A. Basu, G. Blanford, S. Simon, R. Reedy, B. M. French, and J. Papike, The Lunar Regolith, in Lunar Sourcebook, edited by G. H. Heiken, D. T. Vaniman, and B. M. French, 736 pp, Cambridge University Press, New York, 1991.

McKinney, G. W., D. J. Lawrence, T. H. Prettyman, R. C. Elphic, W. C. Feldman, and J. J. Hagerty, MCNPX benchmark for cosmic ray interactions with the Moon, J. Geophys. Res., 111, E06004, 2006.

Prettyman, T. H., J. J. Hagerty, R. C. Elphic, W. C. Feldman, D. J. Lawrence, G. W. McKinney, and D. T. Vaniman, Elemental composition of the lunar surface: Analysis of gamma ray spectroscopy data from Lunar Prospector, J. Geophys. Res., 111, E12007, 2006.

Reedy, R. C., Planetary gamma-ray spectroscopy, Proc. Lunar Planet. Sci. Conf., 9, 2961, 1978.

Reedy, R. C., Nuclide production by primary cosmic-ray protons, J. Geophys Res., 92, E697-E702, 1987.

Reedy, R. C. and S. C. Frankle, Prompt gamma rays from radiative capture of thermal neutrons by elements from hydrogen through zinc, Atomic Data and Nuclear Data Tables, 80, 1-34, 2002.

Reedy, R. C., J. R. Arnold, and J. I. Trombka, Expected $\gamma$ ray emission spectra from the lunar surface as a function of chemical composition, $J$. Geophys Res., 78, 5847-5866, 1973.

Sasaki, S., Y. Iijima, K. Tanaka, M. Kato, M. Hashimoto, H. Mizutani, and Y. Takizawa, The SELENE mission: Goals and status, Adv. Space Res., 31, 2335-2340, 2003.

Shibata, K., T. Kawano, T. Nakagawa, O. Iwamoto, J. Katakura, T. Fukahori, S. Chiba, A. Hasegawa, T. Murata, H. Matsunobu, T. Ohsawa, Y Nakajima, T. Yoshida, A. Zukeran, M. Kawai, M. Baba, M. Ishikawa, T. Asami, T. Watanabe, Y. Watanabe, M. Igashira, N. Yamamuro, H. Kitazawa, N. Yamano, and H. Takano, Japanese Evaluated Nuclear Data Library Version 3 Revision-3: JENDL-3.3, J. Nucl. Sci. Technol., 39, 1125, 2002.

Silberberg, R., C. H. Tsao, and A. F. Barghouty, Updated Partial Cross Sections of Proton-Nucleus Reactions, The Astrophys. J., 501, 911-919, 1998.

Simpson, J. A., Elemental and isotopic composition of the galactic cosmic rays, Annu. Rev. Nucl. Part. Sci., 33, 323-381, 1983.

Taylor, S. R., The Origin of the Moon: Geochemical Considerations, in Origin of the Moon, edited by W. K. Hartmann, R. J. Phillips, and C. J. Taylor, 781 pp, Lunar and Planetary Institute, Houston, 1986.

Yamashita, N., N. Hasebe, T. Miyachi, E. Shibamura, H. Okada, O. Okudaira, H. Sori, T. Koike, T. Hiramoto, A. Nagashima, T. Fukuoka, M. Kase, V. Dmitrenko, and K. Vlasik, Measurements and calculations of gamma rays from proton-irradiated thick targets, Adv. Space Res., 31, 2359-2362, 2003.

Yamashita, N., N. Hasebe, M.-N. Kobayashi, T. Miyachi, M. Miyajima, O. Okudaira, A. A. Berezhnoy, and E. Shibamura, Leakage of gamma rays and neutrons from thick targets bombarded by energetic protons, Proc. Lunar Planet. Sci. Conf., XXXV, 2004a.

Yamashita, N., N. Hasebe, M.-N. Kobayashi, T. Miyachi, O. Okudaira, E. Shibamura, and A. A. Berezhnoy, Gamma Rays from Major Elements by Thermal Neutron Capture Reactions: Experiment and Simulation for Planetary Gamma-Ray Spectroscopy, The 40th Vernadsky/Brown Microsymposium on Comparative Planetology, Moscow, Russia, Abstract \#79, 2004b.

Yamashita, N., N. Hasebe, T. Miyachi, M. Miyajima, K. Sakurai, M. Kobayashi, O. Okudaira, H. Yamamoto, E. Shibamura, Y. Uchihori, and M. Kase, Energy Spectra of Prompt Gamma Rays from Al and Fe Thick Targets Irradiated by Helium and Proton Beams: Concerning Planetary Gamma-Ray Spectroscopy, J. Phys. Soc. Japan, 75, 054201, 2006.

N. Yamashita (e-mail: nao.yamashita@toki.waseda.jp), N. Hasebe, T. Miyachi, M. Kobayashi, O. Okudaira, S. Kobayashi, T. Ishizaki, K. Sakurai, M. Miyajima, R. C. Reedy, C. d'Uston, S. Maurice, and O. Gasnault 